\title{
Intraindividual validation of 4D flow measurement against 2D flow measurements in aortas with bicuspid or tricuspid valves by cardiovascular magnetic resonance (CMR)
}

\author{
Ahmed E Kharabish ${ }^{2^{*}}$, Kristina Belker ${ }^{1}$, Christian Meierhofer ${ }^{1}$, Stefan Martinoff ${ }^{3}$, Peter Ewert ${ }^{1}$, Heiko Stern ${ }^{1}$, \\ Sohrab Fratz ${ }^{1}$
}

From 18th Annual SCMR Scientific Sessions

Nice, France. 4-7 February 2015

\section{Background}

Theoretically, blood flow in the ascending aorta of patients with helical flow patterns, as in patients with bicuspid aortic valves (BAV), are underestimated by routine two dimensional (2D) phase contrast velocity encoding (PC-VENC). Four dimensional (4D) PC-VENC is theoretically not influenced by flow patterns. Hence, both, 2D PC-VENC and 4D PC VENC should result in similar blood flow measurements in the ascending aorta of subjects without helical flow patterns, as in subjects with tricuspid aortic valves (TAV).

\section{Methods}

To test this hypothesis, we determined blood flow in the ascending aorta of sixteen patients with BAV and helical flow and eighteen healthy subjects with TAV and nonhelical flow by 2D PC-VENC and 4D PC-VENC. Each data set was analyzed by two observers blinded to the other results.

\section{Results}

In patients with BAV and helical flow, 4D PC-VENC resulted in systematically higher blood flow volumes than 2D PC-VENC. In subjects with TAV and nonhelical flow, there was no systematical difference between $4 \mathrm{D}$ and 2D PC-VENC.

\section{Conclusions}

Helical flow patterns as in the ascending aorta of patients with BAV may be more correctly quantified by 4D-VENC compared to 2D PC-VENC.

\section{Funding}

All authors have no conflict of interest.

\section{Authors' details}

${ }^{1}$ Pediatric Cardiology and Congenital Heart Defects, German Heart center, Munich, Germany. ${ }^{2}$ Radiology Department, Cairo University, Cairo, Egypt. ${ }^{3}$ Radiology Department, German Heart Center, Munich, Germany.

Published: 3 February 2015

doi:10.1186/1532-429X-17-S1-P216

Cite this article as: Kharabish et al: Intraindividual validation of 4D flow measurement against 2D flow measurements in aortas with bicuspid or tricuspid valves by cardiovascular magnetic resonance (CMR). Journal of Cardiovascular Magnetic Resonance 2015 17(Suppl 1):P216.

${ }^{2}$ Radiology Department, Cairo University, Cairo, Egypt

Full list of author information is available at the end of the article

(c) 2015 Kharabish et al; licensee BioMed Central Ltd. This is an Open Access article distributed under the terms of the Creative Commons Attribution License (http://creativecommons.org/licenses/by/4.0), which permits unrestricted use, distribution, and reproduction in any medium, provided the original work is properly cited. The Creative Commons Public Domain Dedication waiver (http://creativecommons.org/publicdomain/zero/1.0/) applies to the data made available in this article, unless otherwise stated. 


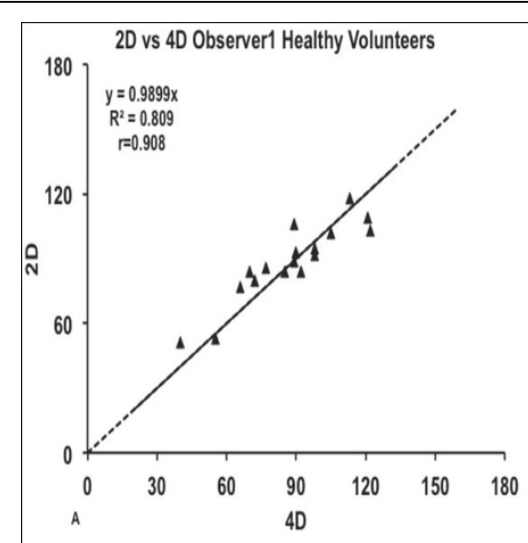

2D vs 4D Observer1 BAV

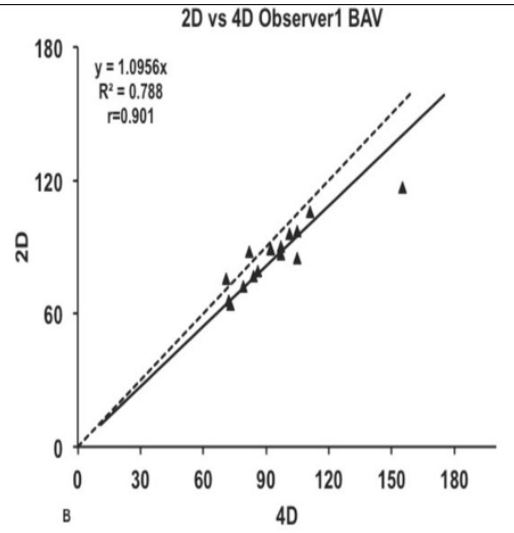

$2 D$ vs $4 D$ Observer2 Healthy Volunteers

$2 D$ vs 4 D Observer2 BAV
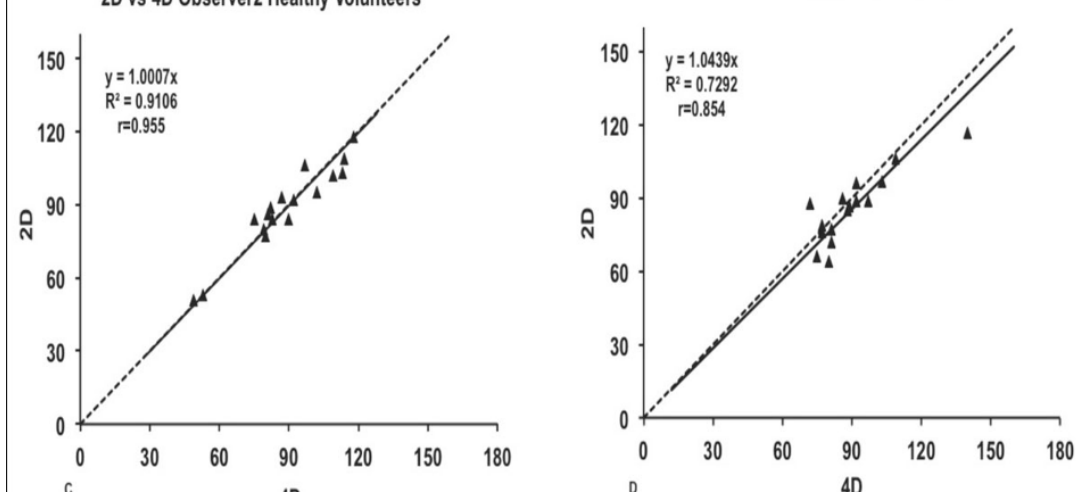

Figure 1 Linear regression correlation. Net flow values $(\mathrm{ml})$ in the ascending aorta of both techniques by the two observers are plotted on both $\mathrm{X}$-axis and $\mathrm{Y}$-axis. The $\mathrm{X}$-axis represents the four-dimensional (4D) method of flow analysis while the $\mathrm{Y}$-axis represents the two-dimensional (2D) method of flow analysis in all four graphs. Graph A, and C represent Health volunteers, B and D represent patients with bicuspid aortic valve (BAV). A line of identity (dashed) $y=x$ is added. The regression line (solid) indicates the offset between the two methods. 